\title{
The role of photosynthesis and amino acid metabolism in the energy status during seed development
}

\author{
Gad Galili $^{1}$ *, Tamar Avin-Wittenberg ${ }^{2}$, Ruthie Angelovici ${ }^{3}$ and Alisdair R. Fernie ${ }^{2}$ \\ ${ }^{1}$ Department of Plant Sciences, The Weizmann Institute of Science, Rehovot, Israel \\ ${ }_{2}^{2}$ Max-Planck-Institut für Molekulare Pflanzenphysiologie, Potsdam-Golm, Germany \\ ${ }^{3}$ Department of Biochemistry and Molecular Biology, Michigan State University, East Lansing, MI, USA
}

\section{Edited by:}

Brian A. Larkins, University of

Nebraska-Lincoln, USA

\section{Reviewed by:}

Alessandro Vitale, Consiglio Nazionale delle Ricerche - National Research

Council of Italy, Italy

Simona Masiero, Universitá degli

Studi di Milano, Italy

\section{*Correspondence:}

Gad Galili, Department of Plant

Sciences, The Weizmann Institute of

Science, 234 Herzl Street,

Rehovot 7610001, Israel

e-mail:gad.galili@weizmann.ac.il

\begin{abstract}
Seeds are the major organs responsible for the evolutionary upkeep of angiosperm plants. Seeds accumulate significant amounts of storage compounds used as nutrients and energy reserves during the initial stages of seed germination. The accumulation of storage compounds requires significant amounts of energy, the generation of which can be limited due to reduced penetration of oxygen and light particularly into the inner parts of seeds. In this review, we discuss the adjustment of seed metabolism to limited energy production resulting from the suboptimal penetration of oxygen into the seed tissues. We also discuss the role of photosynthesis during seed development and its contribution to the energy status of developing seeds. Finally, we describe the contribution of amino acid metabolism to the seed energy status, focusing on the Asp-family pathway that leads to the synthesis and catabolism of Lys, Thr, Met, and Ile.
\end{abstract}

Keywords: seed development, metabolism and bioenergetics, photosynthesis, branched chain amino acids, TCA cycle

\section{INTRODUCTION}

Seeds are the major organ responsible for the evolutionary upkeep of the plant lineage. They store the genetic material of the plant within the embryo, thus guarantying continuation of the plant's life cycle in the next generation. Seed development is highly regulated, with distinct transcript, protein and metabolite switches occurring in a concerted manner throughout its progression (Fait et al., 2006; Sreenivasulu et al., 2008; Xu et al., 2008; Angelovici et al., 2010). Seeds produce a great variety of storage compounds, among them a myriad of carbohydrates (especially starch), storage proteins, and storage lipids. These provide - both directly in the form of food and indirectly in the form of feed $\sim 70 \%$ of the world's human caloric intake (Sreenivasulu and Wobus, 2013). Storage metabolites are subsequently used as nutrient and energy sources to support early stages of seed germination, while the seedling is still heterotrophic. Production of storage compounds whose generation by respiration becomes limiting due to a limitation of oxygen penetration into the dense seed tissues requires a considerable amount of energy. The requirement for oxygen and hence energy production becomes particularly critical during reserve metabolites accumulation and seed desiccation, in which the dense seed tissues limit the oxygen penetration and thus limit mitochondrial energy production (Weber et al., 2005; Angelovici et al., 2010). Photosynthesis in green seeds, and other energy conservation mechanisms, such as a decrease of overall respiration rates during embryo development, somewhat relieve the major drop in energy demand, but do not sufficiently compensate for the energy requirement (Weber et al., 2005; Angelovici et al., 2010). In seeds of the model plant Arabidopsis thaliana, amino acid metabolism is modulated so that many amino acids are synthesized and accumulated during seed desiccation (Fait et al., 2006). In recent years, several lines of evidence have implied that amino acids are not only used for the synthesis of storage proteins, but also, upon energy demand, can be catabolized and their catabolic products fed into the TCA cycle to generate energy (Zhu and Galili, 2004; Angelovici et al., 2010; Galili, 2011). This was also shown for plants exposed to extended darkness, another condition characterized by energy deprivation (Ishizaki et al., 2005, 2006; Araujo et al., 2010). In this mini-review, we describe lines of evidence demonstrating the contribution of photosynthesis and amino acid metabolism to the energy production in developing seeds and the contribution of amino acid catabolism to the energy status of seedlings exposed to extended darkness.

\section{DEVELOPING SEEDS FACE AN EXTREME SHORTAGE OF ENERGY DUE TO LIMITED PENETRATION OF OXYGEN, PARTICULARLY INTO THE INNER SEED PARTS}

Since developing seeds are quite dense, they suffer from limited penetration of oxygen, particularly into the inner seed tissues. The shortage of oxygen extensively limits energy-requiring metabolic processes crucial for seed development and embryo maturation (Vigeolas et al., 2003, 2011; van Dongen et al., 2004). In developing seeds of dicotyledonous (dicot) plant species, photosynthesis in the developing embryo contributes a considerable amount of oxygen to the seed tissue, which further fuels energygenerating biochemical pathways, such as glycolysis and respiration (Rolletschek et al., 2003; Ruuska et al., 2004; Borisjuk et al., 2005; Tschiersch et al., 2011, 2012). This is in contrast to developing non-green seeds of monocotyledonous (monocot) plants that do not generate energy via photosynthesis, and hence must depend on alternative energy-generating metabolic processes in addition to glycolysis and respiration in order to fuel seed development. The chemical donors for energy-generation are hypothetically 
produced in the vegetative parts of the plant, using particularly energy derived from photosynthesis, and are transported to the developing seeds (van Dongen et al., 2004). A number of different metabolites produced in the vegetative tissues are transported to the seeds; however, at a quantitative level, the amino acid Asn, which accumulates to relatively high levels during senescence, is particularly notable as a major energy source (Credali et al., 2013). Its role as an energy donor occurs via the conversion of Asn into Asp, the direct precursor of the branched Asp-family pathway (Figure 1). The Asp-family pathway synthesizes the amino acids Lys, Thr, Met, and Ile, which, under conditions of energy shortage, are further catabolized by the TCA cycle to generate energy (Galili, 2011). An energy shortage does not occur only in seeds, but also in vegetative tissues that lack photosynthesis, and upon exposure of plants to extended darkness. Under these energy shortage conditions, Lys is directly catabolized into the TCA cycle, while Thr and Met are converted into Ile, which is then directly catabolized into the TCA cycle (Joshi et al., 2006; Araujo et al., 2010). We focus in this review on the catabolism of Lys and Ile in respect to the contribution of amino acid catabolism to the energy status either during seed development or upon exposure to extended darkness.

\section{CONTRIBUTION OF SEED PHOTOSYNTHESIS TO OXYGEN AVAILABILITY IN DEVELOPING SEEDS}

A significant amount of information on the role of seed photosynthesis for the synthesis of seed reserve compounds has been gathered at the IPK Institute for Plant Genetics in Gatersleben Germany (http://www.ipk-gatersleben.de/en/), where they analyzed developing soybean seeds, which possess active seed photosynthesis (Rolletschek et al., 2003; Ruuska et al., 2004; Borisjuk et al., 2005; Tschiersch etal., 2011, 2012). Light intensity and oxygen level progressively decrease with a progressive increase in seed depth (Vigeolas et al., 2003). Oxygen level is highest in light illuminated seeds, progressively lower in seeds that are not illuminated and lowest in seeds exposed to darkness. The ATP/ADP ratio is markedly higher in developing seeds exposed to light, indicating that photosynthesis in illuminated seeds contributes to the production of energy (Borisjuk et al., 2005). These results indicate that photosynthesis, which produces oxygen, significantly contributes to the energy status of developing seeds. Interestingly, in an independent study, overexpression of hemoglobin 2 in Arabidopsis seeds improved the energy status of seeds under low oxygen conditions and led to an increase in the fatty acid content of developing and mature seeds, further emphasizing the importance of oxygen availability during seeds development (Vigeolas et al., 2011). Photosynthesis could also be important in relatively large seeds, such as soybean seeds, whose inner cell layers receive significantly lower oxygen levels that the outer seed tissues. Borisjuk etal. (2005) demonstrated that developing dicot seeds with green embryos display a reduction in photosynthetic activity during development, starting from the interior of the embryo. This indicates the contribution of this photosynthetic activity to seed energy status.

Photosynthesis encompasses two different reactions, namely: (i) the light reactions that lead to reduction of NADP to NADPH and create a proton gradient across the chloroplast membrane

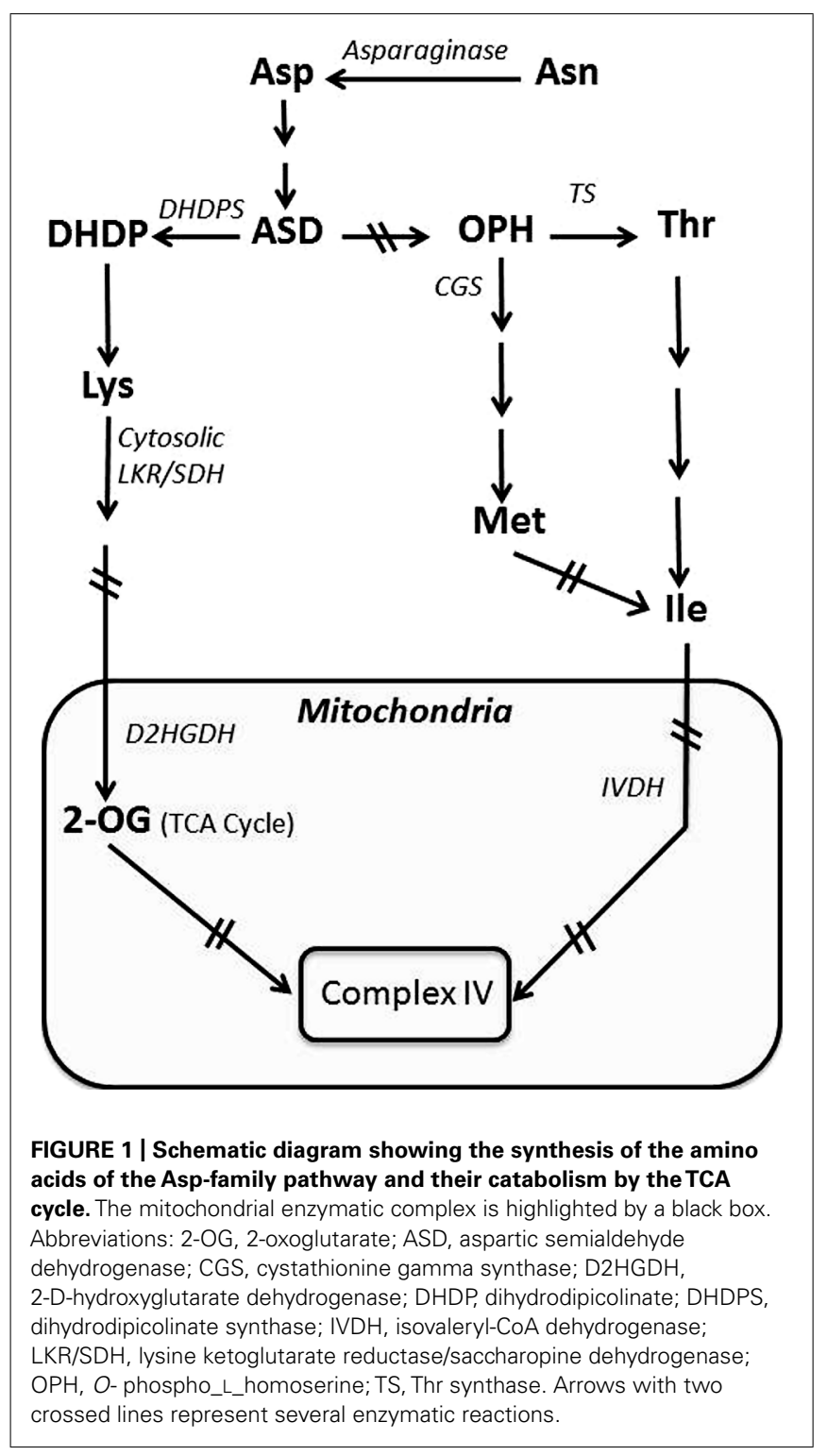

that is used for ATP synthesis; and (ii) the light-independent reactions in which RuBisCO fixes $\mathrm{CO}_{2}$ from the atmosphere in a NADPH-requiring process, and the Calvin-Benson cycle produces sugars (Buchanan et al., 2000). Interestingly, developing oilseed rape (Brassica napus L) embryos possess active photosynthesis, and RuBisCO acts in a special metabolic context without the Calvin-Benson cycle in order to improve the efficiency of carbon utilization during the synthesis of oil storage (Schwender et al., 2004a). This special pathway generates 20\% more acetyl-CoA, a precursor for fatty acid biosynthesis, in comparison to glycolysis and saves $40 \%$ of the carbon that would otherwise be lost as $\mathrm{CO}_{2}$.

\section{CONTRIBUTION OF AMINO ACID METABOLISM TO THE ENERGY STATUS OF DEVELOPING SEEDS}

Amino acids are constituents of proteins and hence essential components for the life of all organisms. Yet, in response to specific 
developmental and stress-associated conditions, amino acids also serve as energy donors through their catabolism in the TCA cycle (Araujo et al., 2010; Angelovici et al., 2011; Kirma et al., 2012). In vegetative tissues, photosynthesis is the major source of energy during daytime, and thus during daytime amino acids are used for the synthesis of proteins and, as precursors of multiple secondary metabolites (Less and Galili, 2008). Yet, during night-time or in response to stresses that cause major energy shortages due to the lack of or reduction in photosynthesis, amino acids also serve as important energy sources through their catabolism via the TCA cycle (Araujo et al., 2011). Therefore, the contributions of amino acid catabolism to the energy status requirements of developing seeds appears even more critical than in vegetative tissues, due to the limits of oxygen diffusion.

\section{CONTRIBUTION OF Lys SYNTHESIS AND CATABOLISM IN THE TCA CYCLE TO SEED ENERGY STATUS}

Insight into the potential importance of Lys catabolism to the energy status of developing seeds was obtained in research aiming to enhance the nutritional quality of seeds by increasing Lys accumulation (Zhu and Galili, 2003). Lys is an essential amino acid that serves a vital role in human food and livestock feed, since humans and some livestock (such as chicken and pigs, i.e., mono-gastric livestock) are unable to synthesize Lys (Kirma et al., 2012). The synthesis of Lys in plants (Figure 1) is subject to post-translational regulation, where increasing Lys feedback inhibits the activity of dihydrodipicolinate synthase (DHDPS), which catalyzies the first committed step in the Lys biosynthetic branch of the Asp-family pathway. Plants possess two DHDPS isozymes (DHDPS1 and DHDPS2), with DHDPS2 accounting for the majority of the total DHDPS activity (Jones-Held et al., 2012). Because Lys is an essential amino acid, an attempt was made to increase Lys in seeds of the model plant Arabidopsis, using a recombinant gene encoding in a seed-specific manner a mutant bacterial DHDPS2 enzyme insensitive to Lys feedback inhibition (Zhu and Galili, 2003). This approach yielded only a relatively mild increase in seed Lys, leading to the hypothesis that this amino acid is not an end product metabolite, but rather might be catabolized in the TCA cycle to generate energy. To test this hypothesis, the same bacterial feedback-insensitive DHDPS was expressed in seeds of a transgenic Arabidopsis genotype that lacks the capacity for Lys catabolism due to a knockout mutation in the gene encoding the bi-functional enzyme lysineketoglutarate reductase/saccharopine dehydrogenase (LKR/SDH), which contains the first two enzymes of Lys catabolism linked as a single polypeptide. In this combined genotype, seed Lys content was nearly 1000-fold higher than wild type (WT), indicating that developing seeds have a strong flux of Lys synthesis as well as catabolism into the TCA cycle (Zhu and Galili, 2003).

Developing seeds possessing significantly enhanced Lys levels due to increased Lys synthesis and suppression of Lys catabolism also exhibited notable differences in their transcriptomes and primary metabolomes, indicating that Lys metabolism (synthesis and catabolism) is well connected to the TCA cycle (Angelovici et al., 2009, 2011). For example, compared to WT, the levels of the TCA cycle metabolites fumarate, citrate, and ketoglutarate are lower in the genotype possessing enhanced Lys synthesis and a block of Lys catabolism, compared to the wild-type, genotype (Angelovici et al., 2009, 2011). Lys catabolism into the TCA cycle is apparently used to generate energy essential for seed development. As shown in Figure 1, Lys is not the only amino acid of the Asp-family pathway that feeds into the TCA cycle. A second branch of the Asp-family pathway leads to the synthesis of Thr and Met, which are further metabolized into Ile, which also feeds into the TCA cycle and serves as a major substrate for energy generation (Joshi et al., 2006; Kochevenko and Fernie, 2011).

In the developing seed, the upstream substrate that feeds the Asp-family pathway on route to the TCA cycle is Asn. Asn is transported from vegetative tissues to developing seeds where it is converted by asparaginase into Asp, the starting point metabolite of the Asp-family pathway (Figure 1; Credali et al., 2013). Indeed, the asparaginase level is generally stimulated during early seed development (Dickson et al., 1992; Credali et al., 2013).

\section{AMINO ACID CATABOLISM FACILITATES RESPIRATION UNDER STRESS CONDITIONS}

Under normal conditions, respiration depends on the oxidation of carbohydrates. However, during situations in which carbohydrate supply is limited, the plant cell can modify its metabolism to utilize alternative respiratory substrates. Among these substrates are proteins. Protein degradation is a highly regulated process, involving a multitude of cellular reactions, such as ubiquitinylation and degradation via the proteasome, the autophagy machinery and nutrient sensing by the TOR pathway. The product of protein degradation, free amino acids, can be further catabolize to generate energy (ATP; Zhu and Galili, 2003; Joshi et al., 2006; Araujo et al., 2011; Kochevenko and Fernie, 2011). Indeed, it has been shown that under abiotic stress there is increased transcription of amino acid catabolic genes (Less and Galili, 2008).

In mammals, the mitochondrial protein, electron-transfer flavoprotein:ubiquinone oxireductase (ETFQO), accepts electrons from electron transfer flavoprotein (ETF) to reduce ubiquinone. ETF serves as an obligatory electron acceptor for nine mitochondrial flavoprotein dehydrogenases. The ETF/ETFQO system facilitates electron transfer from these flavoprotein dehydrogenases to the main respiratory chain (Ishizaki et al., 2005, 2006). Homologs of ETF and ETFQO have been characterized in plants, as well as two flavoprotein dehydrogenases: isovaleryl-CoA dehydrogenase (IVDH) and 2-D-hydroxyglutarate dehydrogenase (D2HGDH; Ishizaki et al., 2005, 2006; Araujo et al., 2010). T-DNA insertion mutants of each of these genes display increased sensitivity to prolonged darkness, a condition that leads to carbon starvation. In addition, metabolic analysis of these mutants under prolonged darkness revealed an accumulation of several amino acids and an intermediate metabolite of Leu catabolism in comparison to WT. These results suggest a role for amino acid catabolism in supplying respiratory intermediates during carbon starvation (Figure 2; Ishizaki et al., 2005, 2006). It has been demonstrated that IVDH is apparently predominantly responsible for the transport of electrons from breakdown products of phytol and branchedchain amino acids (BCAA: Ile, Leu, and Val), while D2HGDH 


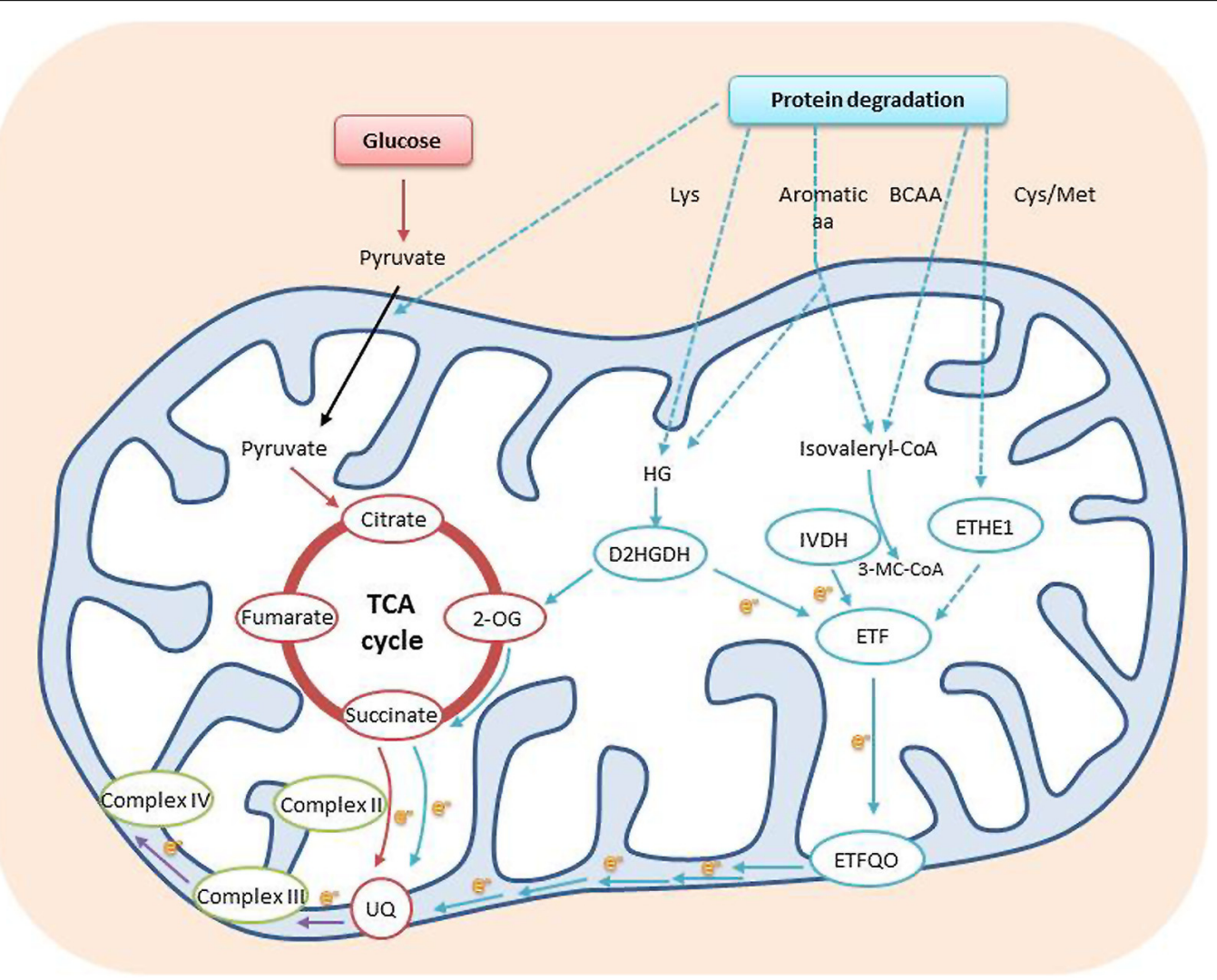

FIGURE 2 |Amino acids can be used as electron donors to the mitochondrial electron transport chain. Protein degradation yields a range of amino acids that are further metabolized either into isovaleryl-CoA or HG. Isovaleryl-CoA can be produced by catabolism of the branched chain and aromatic amino acids and by both phytol and Lys degradation, whereas $\mathrm{HG}$ can be produced by aromatic amino acid degradation or from the Lys derivative L-pipecolate. The electrons generated are transferred to the respiratory chain through to the ubiquinol pool via an ETF/ETFQO system. Possible involvement of sulfur containing amino acids has also been implicated by the phenotype of ETHE1 knockdown plants. Some amino acids can facilitate energy production via the TCA cycle, either by conversion to pyruvic acid or acetyl-CoA or by direct conversion to TCA cycle intermediates, such as 2-OG, and direct electron supply to the ubiquinone pool of the mitochondrial electron transport chain in plants. Dotted arrows represent possible transport processes and multi enzymatic reactions. Abbreviations: BCAA, branched chain amino acids; D2HGDH, 2-D-hydroxyglutarate dehydrogenase; e-, electron; ETF, electron transfer flavoprotein; ETFQO, ETF:ubiquinone oxidoreductase; ETHE1, ethylmalonic encephalopathy protein1; HG, hydroxyglutarate; IVDH, isovaleryl-CoA dehydrogenase; 3-MC-CoA, 3-methylcrotonyl-CoA; 2-OG, 2-oxoglutarate; TCA cycle, tricarboxylic acid cycle; UQ, ubiquinone (Adapted from Araujo etal., 2011; Krüssel etal., 2014). is responsible for the transport of electrons from breakdown products of Lys (Figure 2). This last finding is interesting, as it demonstrates the ability of Lys degradation products to contribute electrons directly to the mitochondrial electron transport chain, as well as supply intermediates to the TCA cycle (Araujo et al., 2010).

Although a direct role for the ETF/ETFQO system in seed development has not yet been demonstrated, considerable experimental evidence has accumulated to support this hypothesis. First, the existence of hypoxic conditions and lack of photosynthesis in some seeds point to the need for alternative electron donors in seeds for the mitochondrial electron transport chain. Secondly, analysis of a mutant with high levels of 12 out of the 20 amino acids in seeds was shown to be IVDH defective, further strengthening the role of this enzyme in the regulation of free amino acid homeostasis during seed development (Gu et al., 2010). Thirdly, a recent publication highlighted the role of BCAT2, a BCAA catabolic enzyme, in establishing the variation of BCAA levels in different Arabidopsis accessions (Angelovici et al., 2013). BCAT2 was shown to localize in the mitochondria, and its expression was shown to increase during seed development, suggesting the involvement of BCAA catabolism in seed development, possibly via the ETF/ETFQO system (Angelovici et al., 2013). Additional evidence suggesting the involvement of the ETF/ETFQO system in seed development was obtained using genetic manipulation of amino acid delivery to pea (Pisum sativum) embryos. When an increase of amino acid delivery was induced, transcripts of the ETF complex were most highly induced (Weigelt et al., 2008). It is important to note that etf and etfqo mutants display lower seed set and shorter siliques when grown under long day conditions (Ishizaki et al., 2005, 2006). This phenotype is attributed to the maternal tissue, as demonstrated by reciprocal crosses of etfqo mutants and WT plants (Ishizaki etal., 2005). Another finding strengthening the role of the ETF/ETFQO system in seed development is 
a recent publication describing ETHE1, a mitochondrial sulfur dioxygenase. ETHE1 is a matrix protein that has been shown to be involved in the detoxification of sulfur compounds derived from sulfur-containing amino acids (Cys and Met). A complete knockout of ETHE1 is embryo lethal and ETHE1 knockdown plants are viable, but present a delay in seed development. Surprisingly, ETHE1 knockdown plants display higher sensitivity to carbon starvation, reminiscent of the ETF/ETFQO system T-DNA mutants (Figure 2) and have been shown to accumulate BCAA under these conditions. These data suggest a possible role for ETHE1 in the ETF/ETFQO system as well as hinting at the importance of the enzyme, per se, during seed development (Krüssel et al., 2014).

In summary, in this review we discussed a number of studies that have enhanced our understanding of the parallel roles of photosynthesis and amino acid-derived respiratory catabolic processes in meeting the demands for normal energy status during seed development. In respect to amino acids, we focused on Lys degradation and highlighted the involvement of the ETF pathway. Technical advances, including improved understanding of sub-cellular compartmentization of metabolism (Sweetlove and Fernie, 2013), alongside mathematical approaches to understand whole plant physiology as well as metabolite-metabolite associations (Toubiana et al., 2012; Grafahrend-Belau et al., 2013) are now available. These will greatly enhance our understanding of these enzymatic processes. Furthermore, metabolite imaging techniques, such as genetically encoded metabolite sensors (Okumoto et al., 2012), nuclear magnetic imaging (Borisjuk et al., 2013), and flux profiling techniques (Schwender et al., 2004b), alongside miniaturization of respiration measurements (Sew et al., 2013), will additionally enhance our understanding of the shifts in plant energy metabolism that occur during the process of seed development. Our potential to metabolically engineer seeds in a highly tailored manner will be radically improved once such information is available and integrated with our current knowledge.

\section{ACKNOWLEDGMENTS}

Our research was supported by grants from Israel Science Foundation; Bi-national Agriculture Research and Development (BARD); Israel Science Foundation; Ministry of Agriculture; Alternative Energy Research Initiation (AERI) Program and ICORE Biofuels Program (GG); Minerva, Alexander von Humboldt and EMBO fellowships (TAW) and by the Max-Planck Society (ARF).

\section{REFERENCES}

Angelovici, R., Fait, A., Fernie, A. R., and Galili, G. (2011). A seed high-lysine trait is negatively associated with the TCA cycle and slows down Arabidopsis seed germination. New Phytol. 189, 148-159. doi: 10.1111/j.1469-8137.2010.03478.x

Angelovici, R., Fait, A., Zhu, X., Szymanski, J., Feldmesser, E., Fernie, A. R., et al. (2009). Deciphering transcriptional and metabolic networks associated with lysine metabolism during Arabidopsis seed development. Plant Physiol. 151, 2058-2072. doi: 10.1104/pp.109.145631

Angelovici, R., Galili, G., Fernie, A. R., and Fait, A. (2010). Seed desiccation: a bridge between maturation and germination. Trends Plant Sci. 15, 211-2118. doi: 10.1016/j.tplants.2010.01.003

Angelovici, R., Lipka, A. E., Deason, N., Gonzalez-Jorge, S., Lin, H., Cepela, J., et al. (2013). Genome-wide analysis of branched-chain amino acid levels in Arabidopsis seeds. Plant Cell 25, 4827-4843. doi: 10.1105/tpc.113.119370
Araujo, W. L., Ishizaki, K., Nunes-Nesi, A., Larson, T. R., Tohge, T., Krahnert, I., et al. (2010). Identification of the 2-hydroxyglutarate and isovaleryl-CoA dehydrogenases as alternative electron donors linking lysine catabolism to the electron transport chain of Arabidopsis mitochondria. Plant Cell 22, 1549-1563. doi: 10.1105/tpc.110.075630

Araujo, W. L., Tohge, T., Ishizaki, K., Leaver, C. J., and Fernie, A. R. (2011). Protein degradation - an alternative respiratory substrate for stressed plants. Trends Plant Sci. 16, 489-498. doi: 10.1016/j.tplants.2011.05.008

Borisjuk, L., Neuberger, T., Schwender, J., Heinzel, N., Sunderhaus, S., Fuchs, J., et al. (2013). Seed architecture shapes embryo metabolism in oilseed rape. Plant Cell 25, 1625-1640. doi: 10.1105/tpc.113.111740

Borisjuk, L., Nguyen, T. H., Neuberger, T., Rutten, T., Tschiersch, H., Claus, B., et al. (2005). Gradients of lipid storage, photosynthesis and plastid differentiation in developing soybean seeds. New Phytol. 167, 761-776. doi: 10.1111/j.14698137.2005.01474.x

Buchanan, B. B., Gruissem, W., and Jones, R. L. (2000). Biochemistry and Molecular Biology of Plants. Rockville, MD: American Society of Plant Physiologists.

Credali, A., Garcia-Calderon, M., Dam, S., Perry, J., Diaz-Quintana, A., Parniske, M., et al. (2013). The $\mathrm{K}^{+}$-dependent asparaginase, NSE1, is crucial for plant growth and seed production in Lotus japonicus. Plant Cell Physiol. 54, 107-118. doi: $10.1093 / \mathrm{pcp} / \mathrm{pcs} 156$

Dickson, J. M., Vincze, E., Grant, M. R., Smith, L. A., Rodber, K. A., Farnden, K. J., et al. (1992). Molecular cloning of the gene encoding developing seed L-asparaginase from Lupinus angustifolius. Plant Mol. Biol. 20, 333-336. doi: 10.1007/BF00014503

Fait, A., Angelovici, R., Less, H., Ohad, I., Urbanczyk-Wochniak, E., Fernie, A. R., et al. (2006). Arabidopsis seed development and germination is associated with temporally distinct metabolic switches. Plant Physiol. 142, 839-854. doi: 10.1104/pp.106.086694

Galili, G. (2011). The aspartate-family pathway of plants: linking production of essential amino acids with energy and stress regulation. Plant Signal. Behav. 6, 192-195. doi: 10.4161/psb.6.2.14425

Grafahrend-Belau, E., Junker, A., Eschenroder, A., Muller, J., Schreiber, F., and Junker, B. H. (2013). Multiscale metabolic modeling: dynamic flux balance analysis on a whole-plant scale. Plant Physiol. 163, 637-647. doi: 10.1104/pp.113.224006

Gu, L., Jones, A. D., and Last, R. L. (2010). Broad connections in the Arabidopsis seed metabolic network revealed by metabolite profiling of an amino acid catabolism mutant. Plant J. 61, 579-590. doi: 10.1111/j.1365-313X.2009.04083.x

Ishizaki, K., Larson, T. R., Schauer, N., Fernie, A. R., Graham, I. A., and Leaver, C. J. (2005). The critical role of Arabidopsis electron-transfer flavoprotein:ubiquinone oxidoreductase during dark-induced starvation. Plant Cell 17, 2587-2600. doi: 10.1105/tpc.105.035162

Ishizaki, K., Schauer, N., Larson, T. R., Graham, I. A., Fernie, A. R., and Leaver, C. J. (2006). The mitochondrial electron transfer flavoprotein complex is essential for survival of Arabidopsis in extended darkness. Plant J. 47, 751-760. doi: 10.1111/j.1365-313X.2006.02826.x

Jones-Held, S., Ambrozevicius, L. P., Campbell, M., Drumheller, B., Harrington, E., and Leustek, T. (2012). Two Arabidopsis thaliana dihydrodipicolinate synthases, DHDPS1 and DHDPS2, are unequally redundant. Funct. Plant Biol. 39, 10581067. doi: 10.1071/Fp12169

Joshi, V., Laubengayer, K. M., Schauer, N., Fernie, A. R., and Jander, G. (2006). Two Arabidopsis threonine aldolases are nonredundant and compete with threonine deaminase for a common substrate pool. Plant Cell 18, 3564-3575. doi: 10.1105/tpc.106.044958

Kirma, M., Araujo, W. L., Fernie, A. R., and Galili, G. (2012). The multifaceted role of aspartate-family amino acids in plant metabolism. J. Exp. Bot. 63, 4995-5001. doi: $10.1093 /$ jxb/ers119

Kochevenko, A., and Fernie, A. R. (2011). The genetic architecture of branchedchain amino acid accumulation in tomato fruits. J. Exp. Bot. 62, 3895-3906. doi: 10.1093/jxb/err091

Krüssel, L., Junemann, J., Wirtz, M., Birke, H., Thornton, J. D., Browning, L. W., et al. (2014). The mitochondrial sulfur dioxygenase ETHE1 is required for amino acid catabolism during carbohydrate starvation and embryo development in Arabidopsis thaliana. Plant Physiol. 165, 92-104. doi: 10.1104/pp.114.239764

Less, H., and Galili, G. (2008). Principal transcriptional programs regulating plant amino acid metabolism in response to abiotic stresses. Plant Physiol. 147, 316-330. doi: $10.1104 /$ pp.108.115733 
Okumoto, S., Jones, A., and Frommer, W. B. (2012). Quantitative imaging with fluorescent biosensors. Annu. Rev. Plant Biol. 63, 663-706. doi: 10.1146/annurevarplant-042110-103745

Rolletschek, H., Weber, H., and Borisjuk, L. (2003). Energy status and its control on embryogenesis of legumes. Embryo photosynthesis contributes to oxygen supply and is coupled to biosynthetic fluxes. Plant Physiol. 132, 1196-1206. doi: $10.1104 / \mathrm{pp} .102 .017376$

Ruuska, S. A., Schwender, J., and Ohlrogge, J. B. (2004). The capacity of green oilseeds to utilize photosynthesis to drive biosynthetic processes. Plant Physiol. 136, 2700-2709. doi: 10.1104/pp.104.047977

Schwender, J., Goffman, F., Ohlrogge, J. B., and Shachar-Hill, Y. (2004a). Rubisco without the Calvin cycle improves the carbon efficiency of developing green seeds. Nature 432, 779-782. doi: 10.1038/nature03145

Schwender, J., Ohlrogge, J., and Shachar-Hill, Y. (2004b). Understanding flux in plant metabolic networks. Curr. Opin. Plant Biol. 7, 309-317. doi: 10.1016/j.pbi.2004.03.016

Sew, Y. S., Stroher, E., Holzmann, C., Huang, S., Taylor, N. L., Jordana, X., et al. (2013). Multiplex micro-respiratory measurements of Arabidopsis tissues. New Phytol. 200, 922-932. doi: 10.1111/nph.12394

Sreenivasulu, N., Usadel, B., Winter, A., Radchuk, V., Scholz, U., Stein, N., et al. (2008). Barley grain maturation and germination: metabolic pathway and regulatory network commonalities and differences highlighted by new MapMan/PageMan profiling tools. Plant Physiol. 146, 1738-1758. doi: 10.1104/pp.107.111781

Sreenivasulu, N., and Wobus, U. (2013). Seed-development programs: a systems biology-based comparison between dicots and monocots. Annu. Rev. Plant Biol. 64, 189-217. doi: 10.1146/annurev-arplant-050312-120215

Sweetlove, L. J., and Fernie, A. R. (2013). The spatial organization of metabolism within the plant cell. Annu. Rev. Plant Biol. 64, 723-746. doi: 10.1146/annurevarplant-050312-120233

Toubiana, D., Semel, Y., Tohge, T., Beleggia, R., Cattivelli, L., Rosental, L., et al. (2012). Metabolic profiling of a mapping population exposes new insights in the regulation of seed metabolism and seed, fruit, and plant relations. PLoS Genet. 8:e1002612. doi: 10.1371/journal.pgen.1002612

Tschiersch, H., Borisjuk, L., Rutten, T., and Rolletschek, H. (2011). Gradients of seed photosynthesis and its role for oxygen balancing. Biosystems 103, 302-308. doi: 10.1016/j.biosystems.2010.08.007

Tschiersch, H., Liebsch, G., Borisjuk, L., Stangelmayer, A., and Rolletschek, H. (2012). An imaging method for oxygen distribution, respiration and photosynthesis at a microscopic level of resolution. New Phytol. 196, 926-936. doi: 10.1111/j.1469-8137.2012.04295.x

van Dongen, J. T., Roeb, G. W., Dautzenberg, M., Froehlich, A., Vigeolas, H., Minchin, P. E., et al. (2004). Phloem import and storage metabolism are highly coordinated by the low oxygen concentrations within developing wheat seeds. Plant Physiol. 135, 1809-1821. doi: 10.1104/pp.104.040980
Vigeolas, H., Huhn, D., and Geigenberger, P. (2011). Nonsymbiotic hemoglobin-2 leads to an elevated energy state and to a combined increase in polyunsaturated fatty acids and total oil content when overexpressed in developing seeds of transgenic Arabidopsis plants. Plant Physiol. 155, 1435-1444. doi: $10.1104 /$ pp.110.166462

Vigeolas, H., Van Dongen, J. T., Waldeck, P., Huhn, D., and Geigenberger, P. (2003). Lipid storage metabolism is limited by the prevailing low oxygen concentrations within developing seeds of oilseed rape. Plant Physiol. 133, 2048-2060. doi: 10.1104/pp.103.031963

Weber, H., Borisjuk, L., and Wobus, U. (2005). Molecular physiology of legume seed development. Annu. Rev. Plant Biol. 56, 253-279. doi: 10.1146/annurev.arplant.56.032604.144201

Weigelt, K., Kuster, H., Radchuk, R., Muller, M., Weichert, H., Fait, A., et al. (2008). Increasing amino acid supply in pea embryos reveals specific interactions of $\mathrm{N}$ and $\mathrm{C}$ metabolism, and highlights the importance of mitochondrial metabolism. Plant J. 55, 909-926. doi: 10.1111/j.1365-313X.2008.03560.x

Xu, S. B., Li, T., Deng, Z. Y., Chong, K., Xue, Y., and Wang, T. (2008). Dynamic proteomic analysis reveals a switch between central carbon metabolism and alcoholic fermentation in rice filling grains. Plant Physiol. 148, 908-925. doi: 10.1104/pp.108.125633

Zhu, X., and Galili, G. (2003). Increased lysine synthesis coupled with a knockout of its catabolism synergistically boosts lysine content and also transregulates the metabolism of other amino acids in Arabidopsis seeds. Plant Cell 15, 845-853. doi: 10.1105/tpc.009647

Zhu, X., and Galili, G. (2004). Lysine metabolism is concurrently regulated by synthesis and catabolism in both reproductive and vegetative tissues. Plant Physiol. 135, 129-136. doi: 10.1104/pp.103.037168

Conflict of Interest Statement: The authors declare that the research was conducted in the absence of any commercial or financial relationships that could be construed as a potential conflict of interest.

Received: 27 June 2014; accepted: 19 August 2014; published online: 03 September 2014.

Citation: Galili G, Avin-Wittenberg T, Angelovici $R$ and Fernie AR (2014) The role of photosynthesis and amino acid metabolism in the energy status during seed development. Front. Plant Sci. 5:447. doi: 10.3389/fpls.2014.00447

This article was submitted to Plant Evolution and Development, a section of the journal Frontiers in Plant Science.

Copyright (C) 2014 Galili, Avin-Wittenberg, Angelovici and Fernie. This is an openaccess article distributed under the terms of the Creative Commons Attribution License (CC BY). The use, distribution or reproduction in other forums is permitted, provided the original author(s) or licensor are credited and that the original publication in this journal is cited, in accordance with accepted academic practice. No use, distribution or reproduction is permitted which does not comply with these terms. 